\title{
Efficient Mathematical Procedural Model for Brain Signal Improvement from Human Brain Sensor Activities
}

\author{
Rajib Chowdhury \\ Department of Computer Science, American International University Bangladesh, Dhaka, Bangladesh \\ Email: rajib.eee092@gmail.com \\ A.F.M. Saifuddin Saif \\ Department of Computer Science, American International University Bangladesh, Dhaka, Bangladesh \\ Email: saif@aiub.edu
}

Received: 23 June 2018; Accepted: 14 September 2018; Published: 08 October 2018

\begin{abstract}
Human brain signals obtained by the human brain sensor electrodes measure the cerebral activities on the human brain. The main aim of our research is to improve the human brain activities based on the human brain signal. The entire procedure contains three steps. The first step is to acquire the brain signal, then develop this brain signal with the proposed method and finally improve the human brain activities with this modified brain signal. The entire procedure will proceed in a proposed Neuroheadset device embedded with necessary sensors using the non-invasive technique. This device will help to acquire the brain signal, modify this signal and improve the brain activities with this modified brain signal. In this research, we illustrated the first two steps like signal acquisition and signal modification. In the experiment, we used Electroencephalogram as an efficient non-invasive signal acquisition technique for acquiring the brain signal and also introduced a proposed method to modify this signal. This method helped to improve the human brain signal using the required times of the iteration process. In the experiment level, several iteration processes have been done to get above $90 \%$ improvement rate of the brainwaves. In this research, the improved signal has been considered based on the generated brain signal in various aspects like human intelligence, memory and also the capability of better feelings.
\end{abstract}

Index Terms - Artificial intelligence, human brain sensor activities, human brain signal, proposed method, proposed neuroheadset device.

\section{INTRODUCTION}

Human brain activities related research works are contributing a most significant role in today's research world. Researchers are doing more research work based on the human brain activities. The human brain activities are mainly measured by the generated brain signal. In earlier, more research work like recognize of EEG-based emotion, effects of physical exercise, improvement of human brain signal classification, exploration of prominent frequency wave, control of wheelchair, etc. have done using the human brainwaves signal.

Human brain EEG sensors have facilitated to recognize the emotion [1] using the optimal feature selection and the deep learning ensembles method. Support vector machine (SVM) has also been [2] considered for EEG based emotion recognition. Deep Learning Network with covariate shift adaptation based on a principal component has been [3] used for the EEG-Based emotion recognition. Brain-Computer Interface (BCI) has helped to [4] improve the emotion recognition by combining the EEG feature selection and kernel classifiers. The effects [5] of a severe physical exercise based on the individual resting state EEG alpha peak frequency (iAPF) have examined using the EEG activity. Active Learning (AL) has been [6] used for improving the classification of the neural signal in a paradigm of rapid serial visual presentation (RSVP). The EEG wave of prominent frequency has been [7] explored from the network of brain sensors. EEG signal has also been [8] used to control the wheelchair with the help of design and the implementation of the braincomputer interface. It has also [9] investigated that the physiological signal based on the neural and non-neural basis has united to gather decision accuracy and selfassurance of visual and auditory subtasks performed during an executed crew-station experiment.

At present, the major challenge is to improve the human brain activities based on human brain signal which has addressed as a core investigation by proposing a new method in our research. Later aims will add to establish a proposed neuroheadset device for acquiring brain signal, controlling brain signal, developing the brain signal, improving the brain activities based on modified human brain signal by using a non-invasive technique. In this research, we discussed the acquisition of brain signal and the improvement of acquired brain signal. In the future, we will discuss the methodology for improving 
the brain activity using the modified human brain signal and about the proposed neuroheadset device. In this research, we have acquired the EEG-based brain signal using non-invasive signal acquisition technique and also demonstrated the proposed method to improve the brain signal with necessary iteration process. After the acquisition of brain signal, we digitized, pre-processed, and extracted features of the brain signal. In next, we applied the proposed method for improving the brain signal. In this research, analog to digital converter (ADC) helped to increase the signal strength. We have considered independent component analysis (ICA) in the level of signal pre-processing. Fast Fourier transform (FFT) was used to extract features from the pre-processed signal. Then, we also represented the signal data into 256 data points. Our proposed method helped to improve the brain signal by comparing with the data points of the desired signal for every place with the necessary iteration process. The details have been described in section III. In the experiment level, we got more than ninety percent improvement rate for all participated human subjects.

\section{RELATED WORKS}

In [2] the year 2015 Raja Majid Mehmood and Hyo Jong Lee recognized electroencephalogram (EEG) signal based emotion using the Hjorth parameters and also the SVM classifier. In their research, they used a 10/20 internationally recognized placement system for the brain signal acquisition. Then, they used an easy cap on the Brain Vision System with eighteen electrodes for recording the acquired EEG signals. IAPS database has been used to select the different types of emotional stimuli. After recording the EEG signal, EEGLAB toolbox has used for pre-processing the signals. Then Hjorth parameters were computed in the time-frequency domain using these pre-processed signals. For the feature extraction, three Hjorth parameters have been used from the brain lobe and the selected frequency wave. Finally, the SVM classifier was used to classify the emotions.

In [10] the year 2015 Muhammad N. Fakhruzzaman, Edwin Riksakomara and Hatma Suryotrisongko identified the EEG based signal from the human brain activities with the help of EMOTIV EPOC for the motor imagery. In the data acquisition level, they used only one human subject for avoiding the different types of brain signals. In their research, right foot and left-hand moving have been considered as the activities of training. Automatic CSP filter generation has also been included in this step through one of the circumstances inside the chain. Classifier Trainer with the acquirer of input data has been introduced by the executed of next scenario. The scenario helped to read through the data when the feature vectors were extracted and then wrote all of these into one configuration file for further utilize. Support Vector Machine (SVM) has been considered in their research for the classification. In the Open Vibe, mi-csp-4-online.xml has run for executing the step of testing with the help of DSP filter. The entire testing step has been performed of
10 epochs per activity within thirty seconds per session with a five-second time-frame.

In [3] the year 2014 Suwicha Jirayucharoensak, Setha Pan-Ngum and Pasin Israsena recognized the EEG signal based emotion using the deep learning network with principle component-based covariate shift adaption (CSA). In their research, each layer has constituted with a hundred nodes. All of the input features of these nodes have calculated by using the process of feature extraction. Two hundred thirty input features composing of the power spectral density has learned by the system at each time. The principal component analysis (PCA) was used to decrease the problem of over-fitting of the DLN. The most necessary fifty components have extracted by the PCA from the first two hundred thirty input features. In each layer, DLN with fifty concealed nodes fed these extracted features. In the final step, the effectiveness of the emotion recognition system has been developed with the help of CSA to solve the problem of non-stationary in EEG signals. Here, each of the individual input features has normalized with the help of the average of preceding feature significance within a rectangular window. A leave-one-out cross-validation scheme helped to measure the accuracy level of classification of both dimensional states like valence and arousal states for the four experimental steps. In their research, the leave-one-out cross-validation has performed for the thirty-two subject acquisitions. All input features based on other thirty-one subjects helped to composite a training dataset. The power spectral features have been consisted of each dataset from the recording of the EEG signal when subjects have watched the music videos. Here, based on the gradient descent method, the DLN performed its initial bias and weight optimization to get the classification accuracy. The measurement of the accuracy of classification has counted five times. Then the average accuracy has been considered for the further analysis. At next, the hidden nodes of each layer have decreased to fifty nodes for avoiding the effects of performance accuracy. The Nonlinear Principal Component Analysis (NPCA) has performed by the auto-encoder when the visible nodes have greater than hidden nodes. The PCA has been used to help the stack of the auto-encoder for learning the linear correlations between the features of input. LIBSVM tools helped to measure the SVM classifier performance. Naïve Bayes (NB) has also been used in WEKA tool for performing the classification of emotion.

In [11] November 2014 Norizam Sulaiman, Cheng Chee Hau, Amran Abdul Hadi, Mahfuzah Mustafa, and Shawal Jadin established the human thought interpretation with the help of EEG waves and Lab VIEW. In their research, they have used the EMOTIV EPOC EEG amplifier and electrodes for acquiring the EEG waves from the subjects. The location of the fourteen measurement points of EEG covered entire brain lobes like Temporal, Frontal, Occipital, and Parietal. The protocol of the measurement has consisted of five different steps including Move forward, Move backward, Turn right, Turn left, and Stop. The Bluetooth device was 
used to send the EEG signals to the computer and then stored in a file. At next, Lab View has been used to analyze and find the features of EEG. To find out the EEG features for the thinking types, the spectral analysis of the EEG waves has performed in Lab View. The features vector has also determined from the EEG power spectrum ratio and the number of subjects.

In [16] October 2016 Jalal Karam, Salah Al Majeed, Christofer N. Yalung and Lela Mirtskhulava established the identification of brainwaves signal using the neural network. In their research, they constructed a Radial Basis Functions (RBF) Artificial Neural Network (ANN) and implemented a BCI using a NeuroSkys EEG biosensor for the recognition from the brain signals. The two hundred fifty-six data points have been considered for each thought. In here, Bluetooth helped to send the data for the MATLAB documentation. The highest rate of recognition has been considered for "stop" and "backward" with seventy-seven percent and seventy-four percent respectively. Generally, this contribution helped to increase the machine operation performance by primarily identifying the signal in advance of transferring it as a control to the machine.

\section{Proposed ReSEARCH Methodology}

Figure 1 has demonstrated the overall framework diagram for improving the human brain signal with the proposed method. The methodology has described below with the following necessary steps.

\section{A. Signal Acquisition}

For the signal acquisition, five male persons with the age level of 25-30 years old have participated as subjects in this experiment. In this research, Non-Invasive signal acquisition method like [12] Electroencephalogram (EEG) has been considered for acquiring the brain signal. EEG has [13] a high temporal resolution. The EEG signal can also be [18] able to investigate of human brain situation and detect the overall performance ability of the human. $10 / 20$ internally recognized placement system was [2] used to collect the EEG signals. Then, an Easy Cap of Brain Vision System with 18 electrodes was considered to record the EEG signals. After that, analog to digital converter (ADC) was used to digitize the low strength signal. Acquired EEG based signals have been considered for further pre-processing after completing the signal digitization.

\section{B. Signal Preprocessing}

In the step of signal pre-processing, Independent Component Analysis (ICA) has been chosen for reducing the noise and artifacts from the signals because ICA [13] performs better results than PCA and also considered as computationally efficient. ICA also [14] contributes a most vital role in decomposing mixed data and can [17] able to find the components whose temporal patterns of the activities are statistically independent of one another.

\section{Feature Extraction}

After completing the signal pre-processing, necessary features have been extracted from the pre-processed signals. In this research, Fast Fourier transformation (FFT) has [13] been considered as a significant method for the analysis of frequency. FFT also [15] helps to extract the values of poor signal quality.

\section{Proposed Approach}

The Proposed Approach is a two-phase model which can use for improving the performance level of any activities. The main aim of this research is to develop the human brain signal with this proposed approach. The system procedural model and the mathematical procedural model have described in below:

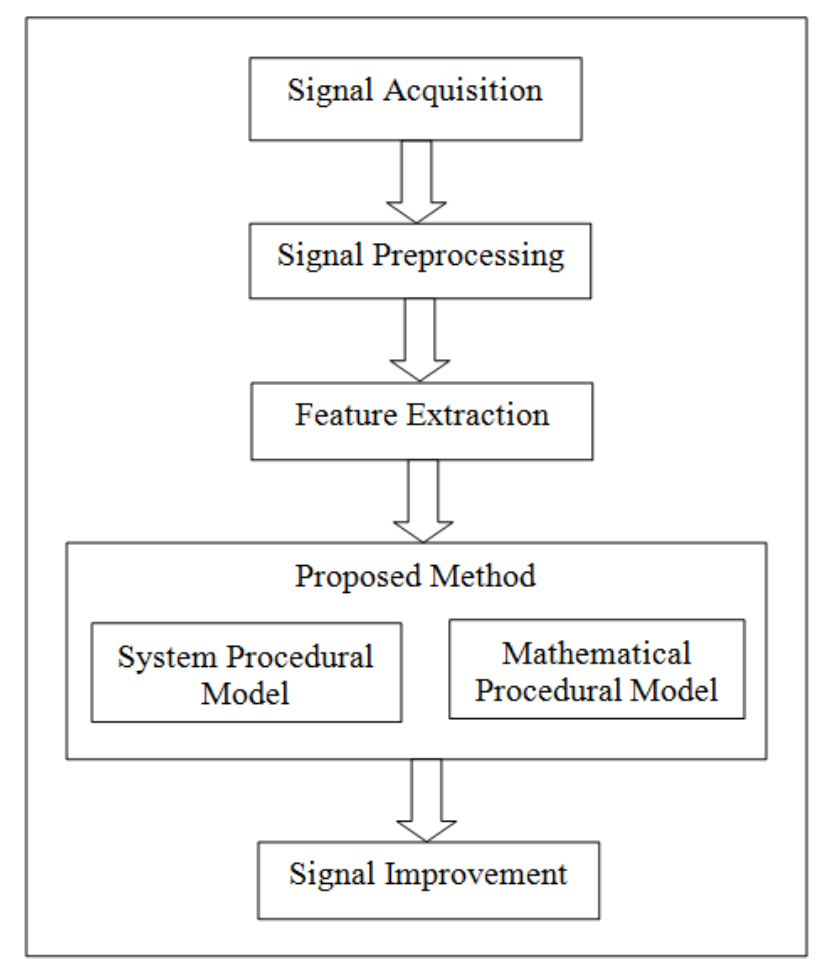

Fig.1. Framework for improving the human brain signal

\section{1) System Procedural Model}

In this model, we have described the overall procedure of the system for improving the EEG based human brain signal with this proposed method. This proposed method helped to improve the human brain signal from the human brain activities. In this phase, at first, 256 data points have been considered based on the feature extracted human brain signal data. Our desired signal data has also converted into 256 data points for the same places as the acquired 256 data points of human brain signal. Then the 256 data of acquired brain signal has compared with the 256 data of desired improved signal in every iteration process. If the input data points greater than the output data points, then the input data will decrease by one in every iteration process until the match 
with the desired output data. If the acquired signal data for a particular place less than the desired improved signal data for that place, then the data of the input signal of that place will increase by one in every iteration process. If the acquired signal data for a particular place equal to the desired improved signal data for that place, then the data of the input signal of that place will remain unchanged. The iteration will stop while the acquired signal data closely related to the desired signal data. The improvement percentage will count after completing the every iteration process.

\section{2) Mathematical Procedural Model}

In this model, we have demonstrated the overall mathematical procedural model for improving the human brain signal. At first, we converted the feature extracted acquired signal data into two hundred fifty-six data point as input,

$$
M_{i}=\left[a_{n}\right]=\left[a_{0}, a_{1}, a_{2}, \ldots . ., a_{255}\right]
$$

Then, we converted the desired output signal data into 256 data point, like the same places of input signal data, as output,

$$
M_{o}=\left[b_{n}\right]=\left[b_{0}, b_{1}, b_{2}, \ldots . ., b_{255}\right]
$$

After that, we have applied below three equations for every input signal data point with the required $I_{S}$ iteration to get the desired improved signal data point.

If the input signals data point same as the output signals data point then input signal data will remain at the same value.

$$
M_{o_{S}}=\left[a_{n}\right] \text { if }\left[a_{n}\right]=\left[b_{n}\right]
$$

If the input signals data greater than output signals data then the input signal data will decrease by 1 in the every iteration process until the match with the desired output signal data.

$$
M_{o_{S}}=\left[a_{n}-1\right] \text { if }\left[a_{n}\right]>\left[b_{n}\right]
$$

If the input signals data less than the output signals data then input signal data will increase by 1 in the every iteration process until the match with desired output signal data.

$$
M_{o_{S}}=\left[a_{n}+1\right] \text { if }\left[a_{n}\right]<\left[b_{n}\right]
$$

Where,

$$
\begin{gathered}
S=[1,2,3,4, \ldots . .] \\
n=[0,1,2,3, \ldots . ., 255]
\end{gathered}
$$

The iteration process will continue until all the input signal data meet with all the desired signal data. When the input signal data match with the desired signal data, then the iteration process will stop.

\section{EXPERIMENTAL RESULTS AND DISCUSSIONS}

In this section, we have discussed the three parts like the experimental setup, experimental results, analysis and discussion.

\section{A. Experimental Setup}

In the experimental setup, at first five male human subjects with the age level of twenty-five to thirty years old have attended for this experiment in our experimental zone. Then an EEG-based cap has been placed in every participant's head one by one. After that, we have acquired EEG-based signal data for all participants one by one. In this experiment, the $10 / 20$ internally recognized placement system was used to collect the EEG signal data. An Easy Cap of Brain Vision System with eighteen electrodes was used to record the EEG signal data. Analog to Digital Converter (ADC) was also used to digitize the brain signal data. After completing the step of digitization for developing the low strength signals, EEG based signals have been considered for further pre-processing steps. Independent Component Analysis (ICA) was chosen to reduce the noise and artifacts from the signals in the signal pre-processing level. Fast Fourier transformation (FFT) was used to extract the features of the pre-processed signal. Then we applied the proposed method for improving the brain signal data. After completing the feature extraction, the brain signal data has converted into 256 data points. The desired output signal data has also converted into two hundred fifty-six data points as like as the same place of input signal data points. Then the all 256 input data points have been changed based on the comparison with all two hundred fifty-six output signal data points in every iteration process. The input signal data points have remained the same value when the input signal data points have the same as the output signal data points. The input signal data point has decreased by one when the input signal data point has greater than the output data point. The input signal data point has also increased by one while the input data point was less than the output signal data point. The iteration process has stopped when the input data points have matched with the desired output data points. The proposed method has also validated using the MATLAB software for the experimental evaluation and analysis.

\section{B. Experimental Results}

In the experimental result, the overall percentages of the improvement rate for the acquired human brain signal of the participated five male human subjects have presented in Table 1. From this table, it has demonstrated 
that the improvement rates are $97.29 \%, 96.09 \%, 98.98 \%$, $90.27 \%$, and $95.33 \%$ for all the subjects respectively using the proposed method. The required iteration processes have also demonstrated in Table 1 for every participant with the improvement rate. In here, 70, 84, 100, 139 and 80 times iterations have been required for getting the improvement rate $97.29 \%, 96.09 \%, 98.98 \%$, $90.27 \%$, and $95.33 \%$ for all the subjects respectively using the proposed method. Figure 2 has also illustrated these improvement rates of that human brain signals for required iteration process. Required iteration processes for getting these overall improvement rates of participated brain signal were seventy, eighty-four, one hundred, one hundred thirty-nine and eighty times for all the subjects respectively as shown in Table 1 . The results have demonstrated that our proposed method helped to get this significant improvement rate of human brain signal.

Table 1. Overall percentage of improvement of the human brain signal with required iteration using proposed approach

\begin{tabular}{|c|c|c|}
\hline Subject & $\begin{array}{c}\text { Improvement Rate (\%) } \\
\text { of the Brain Signal with } \\
\text { Proposed Approach }\end{array}$ & $\begin{array}{c}\text { Required Iteration } \\
\text { Process (times) }\end{array}$ \\
\hline Subject 1 & 97.29 & 70 \\
\hline Subject 2 & 96.09 & 84 \\
\hline Subject 3 & 98.98 & 100 \\
\hline Subject 4 & 90.27 & 139 \\
\hline Subject 5 & 95.33 & 80 \\
\hline
\end{tabular}

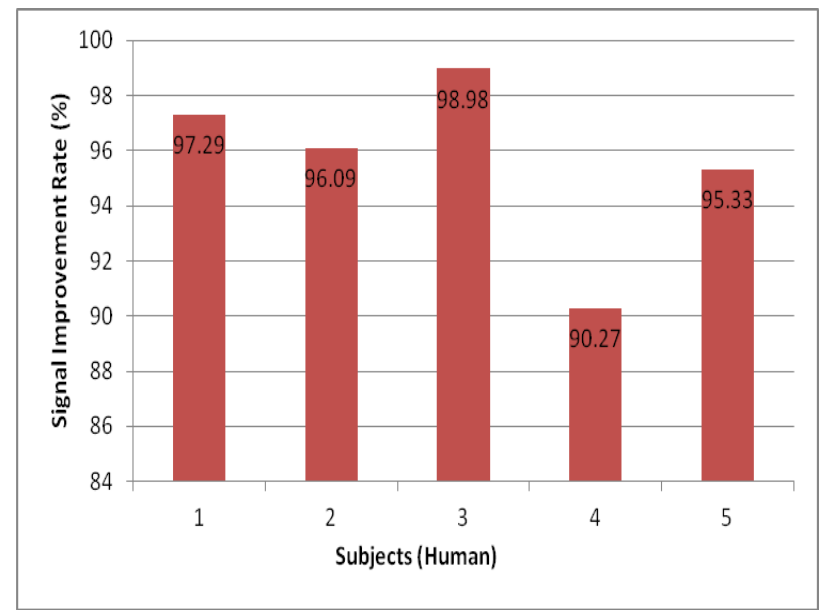

Fig.2. Improvement percentage rate for five participated human brain signals

The necessary of the iteration process for achieving fifty percent, eighty percent and ninety percent improvement rate as improving the human brain signal based on desired output signal with the help of the proposed approach has described in Table 2. From this table, it has demonstrated that the required iteration processes for achieving the fifty percent improvement rate with the proposed method are twelve, seventeen, fifteen, thirty and fifteen times for all the subjects respectively. The required iteration processes for also achieving the eighty percent improvement rate with the proposed approach are thirty-three, forty-two, thirtyseven, eighty-seven and forty times for all the subjects respectively. The required iteration processes for also achieving the ninety percent improvement rate with the proposed approach are forty-eight, sixty-three, fifty-two, one hundred thirty-nine and sixty-two times for all the subjects respectively. In this experiment, we have got the highest level performance of $98.98 \%$ improvement rate for the human brain signal of the participated human subject 3 with the required one hundred times iteration process. In this research, we have got above ninety percent improvement rate for the participated human brain signal with the help of the proposed method. In this experiment, the highest one hundred thirty-nine iteration processes have been required to get the ninety percent improvement rate on the signal data of the subject 3 . From the overall experimental results, we have observed that the several iteration processes will need for getting the improvement rate of the human brain signal data. The iteration process will continue to reach the acquired brain signal data into the desired improved signal data. The necessary of the iteration process will also depend on the generated human brain signal data. The iteration processes will stop while the acquired human brain signal data will closely relate to the desired improved brain signal data.

Table 2. Necessary of iteration process for getting $50 \%, 80 \%$ and $90 \%$ improvement rate of the human brain signals with proposed approach

\begin{tabular}{|c|c|c|c|}
\hline Subject & $\begin{array}{c}\text { Required } \\
\text { Iteration Process } \\
\text { (times) for } \\
\text { achieving 50\% } \\
\text { Improvement } \\
\text { Rate with } \\
\text { Proposed } \\
\text { Approach }\end{array}$ & $\begin{array}{c}\text { Required } \\
\text { Iteration } \\
\text { Process (times) } \\
\text { for achieving } \\
80 \%\end{array}$ & $\begin{array}{c}\text { Required } \\
\text { Iteration } \\
\text { Process (times) } \\
\text { for achieving } \\
\text { Rate with } \\
\text { Proposed } \\
\text { Approach }\end{array}$ \\
$\begin{array}{c}\text { Improvement } \\
\text { Rate with } \\
\text { Proposed } \\
\text { Approach }\end{array}$ \\
\hline Subject 1 & 12 & 33 & 48 \\
\hline Subject 2 3 & 17 & 42 & 63 \\
\hline Subject 4 & 30 & 37 & 52 \\
\hline Subject 5 & 15 & 40 & 62 \\
\hline
\end{tabular}

\section{Analysis and Discussion}

After the result analysis, we have demonstrated that our proposed method was highly performed to acquire more than ninety percent performance of improvement rate with required iteration process. The highest percentage of improvement rate is $98.98 \%$ using our proposed method for the brain signal of subject 3 with one hundred iterations. The required maximum iteration process was one hundred thirty-nine to get $90.27 \%$ improvement rate shown in Table 1 . In Table 2, we described the required iteration process for getting $50 \%$, $80 \%$ and $90 \%$ improvement rate with our proposed method. Figure 3 has also explained the necessary iteration process to get the $50 \%, 80 \%$ and $90 \%$ improvement rate of participated brain signal. From the experimental result, it has demonstrated that the 
necessary of the iteration process will depend on the acquired human brain signal.

In earlier, more research works have been done based on the acquired brain signal with the help of existing methods. The highest percentages of existing research and this research have described in Table 3. In this table, it has demonstrated that the [16] highest recognition rate for brainwaves with radial basis functions (RBF) artificial neural network (ANN) is $77 \%$. RBFs are generally [21] embedded into a feed-forward neural network with two layers that able to classify and model the artificial data. The highest [10] performance of success rate for the movement testing scenario using a support vector machine (SVM) classifier is $91.67 \%$. The maximum emotion [2] recognition rate is $70 \%$ using brainwaves with the SVM classifier. Support vector machine (SVM) is a $[19,20]$ powerful classifier and is also able to give the better classification accuracy than others. The highest improvement rate is $98.98 \%$ for brainwaves of the participated human brain activities with our proposed approach in this research as shown in Table 3. Figure 4 has also shown the highest level percentages of existing research and this research based on the human brainwaves. This graph has illustrated the highest recognition rate of seventy-seven percent, the highest success rate is $91.67 \%$, and the highest emotion recognition rate is $70 \%$ based on previous researches and highest improvement rate is $98.98 \%$ based on this research using human brain signal. Radial basis functions (RBF) artificial neural network (ANN) used to get the brain signal recognition rate. Support vector machine (SVM) used to get the success rate and emotion recognition rate. In this paper, the proposed method has introduced for achieving improvement rate of human brain signal. Table 3 has briefly illustrated the maximum performance based on the previous researches and this research.

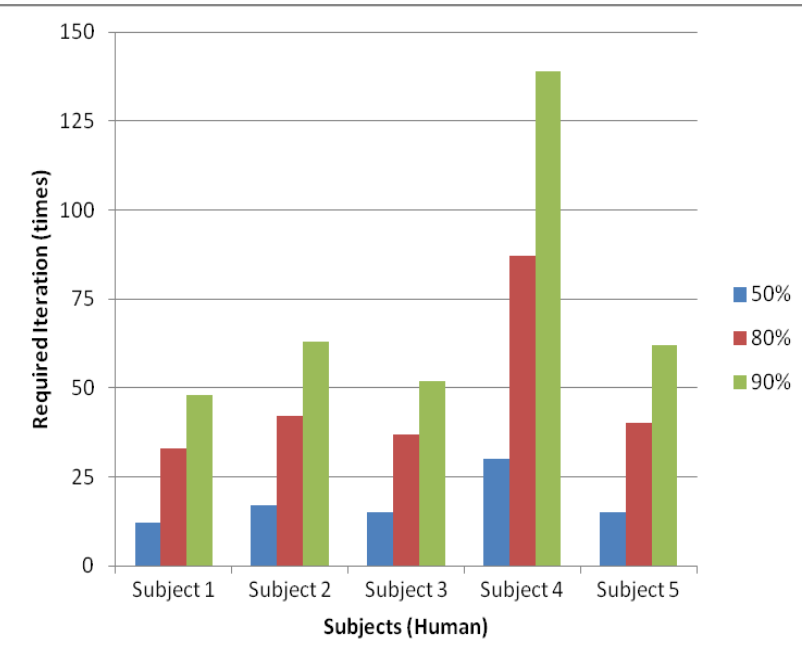

Fig.3. Necessary of iteration processes for getting 50\%, $80 \%$ and $90 \%$ brain signal improvement rate
Table 3. Chart of the highest percentage of this research with proposed approach and the previous researches with existing methods based on human brain waves.

\begin{tabular}{|c|c|c|c|c|}
\hline \multirow[t]{2}{*}{ Subject } & \multicolumn{3}{|c|}{ Based on Previous Researches } & Based on \\
\hline & $\begin{array}{c}\text { The } \\
\text { Highest } \\
\text { Recognition } \\
\text { Rate (\%) of } \\
\text { Brain Wave } \\
\text { Signals } \\
\text { with Radial } \\
\text { Basis } \\
\text { Functions } \\
\text { (RBF) } \\
\text { Artificial } \\
\text { Neural } \\
\text { Network } \\
\text { (ANN) }\end{array}$ & $\begin{array}{c}\text { The } \\
\text { Highest } \\
\text { Success } \\
\text { Rate (\%) } \\
\text { for the } \\
\text { Movement } \\
\text { Testing } \\
\text { Scenario } \\
\text { using } \\
\text { SVM } \\
\text { Classifier }\end{array}$ & $\begin{array}{c}\text { The } \\
\text { Highest } \\
\text { Emotion } \\
\text { Recognition } \\
\text { Rate (\%) } \\
\text { using Brain } \\
\text { Wave } \\
\text { Signals } \\
\text { Using SVM } \\
\text { Classifier }\end{array}$ & $\begin{array}{c}\text { The Highest } \\
\text { Improvement } \\
\text { Rate }(\%) \text { of } \\
\text { Brain Wave } \\
\text { Signals with } \\
\text { Proposed } \\
\text { Approach }\end{array}$ \\
\hline Human & 77.00 & 91.67 & 70.00 & 98.98 \\
\hline
\end{tabular}

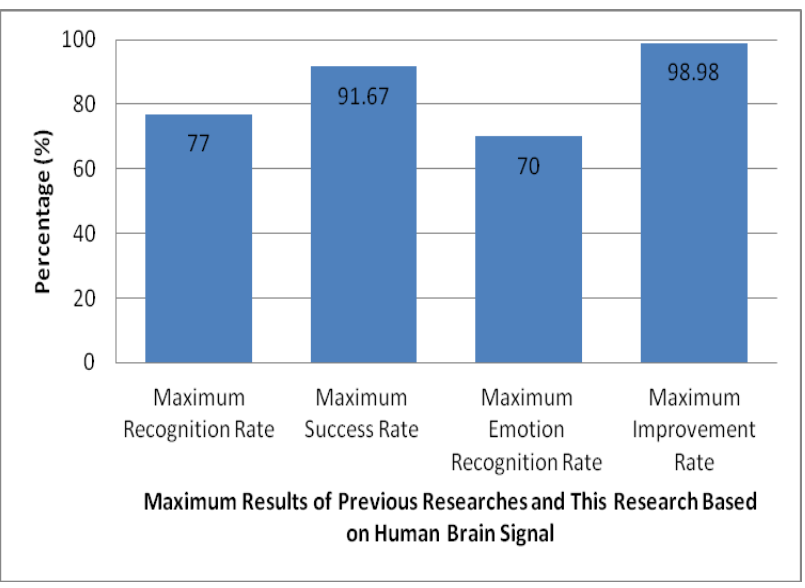

Fig.4. Highest results of previous researches with existing methods and this research with proposed method based on human brain signal

\section{CONCLUSION}

The most significant issue of our research is to improve the human brain activities based on human brainwaves using the proposed method. The overall process for improving human brain activities has divided into following three steps. The first step is to get the human brain signal based on human brain activities, then improve this brain signal by change with the proposed method and finally use this modified brain signal for improving human brain activities with the proposed neuroheadset device. This proposed neuroheadset device will help to get the brain signal, improve this acquired signal and improve the human brain activity using this modified signal of that human brain with the help of necessary iteration process. In this paper, we have explained the first two steps like acquiring the EEGbased human brain signal and improving the EEG-based 
human brain signal. We have also described a proposed method to improve the EEG-based human brain signal. In the experiment level, our proposed method helped to get above ninety percent improvement rate for all the participated humans. The highest $98.98 \%$ improvement rate has got for the subject 3 with 100 iteration process. In here, the required iteration processes were dependent on generated brain signal. Our proposed method will also help to improve the performance level of any kinds of activities and will also be able to make the highest level of performance of improvement. The proposed method has expected to act as supreme innovation to improve human brain signal in computer vision and artificial research domain.

\section{REFERENCES}

[1] Raja Majid Mehmood, Ruoyu Du and Hyo Jong Lee. Optimal feature selection and deep learning ensembles method for emotion recognition from human brain EEG sensors. IEEE Access, 2017, 5 (99):1-1.

[2] Raja Majid Mehmood and Hyo Jong Lee. EEG based emotion recognition from human brain using Hjorth parameters and SVM. International Journal of BioScience and Bio-Technology, 2015, 7 (3):23-32.

[3] Suwicha Jirayucharoensak, Setha Pan-Ngum, and Pasin Israsena. EEG-based emotion recognition using deep learning network with principal component based covariate shift adaptation. Hindawi Publishing Corporation, The Scientific World Journal, 2014, Volume 2014, Article ID 627892.

[4] John Atkinsona and Daniel Campos. Improving BCIbased emotion recognition by combining EEG feature selection and Kernel classifiers. Expert Systems with Applications: An International Journal, 2016, Volume 47, Issue C, 35-41.

[5] Boris Gutmann, Andreas Mierau, Thorben Hülsdünker, Carolin Hildebrand, Axel Przyklenk, Wildor Hollmann, and Heiko Klaus Strüder. Effects of physical exercise on individual resting state EEG alpha peak frequency. Hindawi Publishing Corporation, Neural Plasticity, 2015, Volume 2015, Article ID 717312.

[6] Amar R. Marathe, Vernon J. Lawhern, Dongrui Wu, David Slayback, and Brent J. Lance. Improved neural signal classification in a rapid serial visual presentation task using active learning. IEEE Trans. on Neural Systems and Rehabilitation Engineering, 2016, 24 (3):333-343.

[7] Raja Majid Mehmood and Hyo Jong Lee. Exploration of prominent frequency wave in EEG signals from brain sensors network. International Journal of Distributed Sensor Networks, 2015, Volume 2015, Article ID 386057.

[8] Rajeshree Mahendra Patil, D.M. Kate and A.P. Thakare. Design and implementation of brain computer interface for wheelchair control. International Research Journal of Engineering and Technology (IRJET), 2016, Volume: 03, Issue 02.

[9] Victor Shih, Ludan Zhang, Christian Kothe, Scott Makeig and Paul Sajda. Predicting decision accuracy and certainty in complex brain-machine interactions. IEEE International Conference on Systems, Man, and Cybernetics (SMC) 2016.

[10] Muhammad N. Fakhruzzaman, Edwin Riksakomara, and Hatma Suryotrisongko. EEG wave identification in human brain with EMOTIV EPOC for motor imagery. Science Direct, Procedia Computer Science, 72 (2015):269 - 276.
[11] Norizam Sulaiman, Cheng Chee Hau, Amran Abdul Hadi, Mahfuzah Mustafa and Shawal Jadin. Interpretation of human thought using EEG signals and Lab VIEW. IEEE International Conference on Control System, Computing and Engineering, 2014, 28 - 30, Penang, Malaysia.

[12] T. Kameswara Rao, M. Rajyalakshmi and T. V. Prasad. An exploration on brain computer interface and its recent trends. International Journal of Advanced Research in Artificial Intelligence (IJARAI), 2012, 1 (8):17-22.

[13] M. Rajya Lakshmi, T. V. Prasad and V. Chandra Prakash. Survey on EEG signal processing methods. International Journal of Advanced Research in Computer Science and Software Engineering, 2014, Volume 4, Issue 1, January 2014 ISSN: 2277 128X.

[14] Chuang Li, Han Yuan, Diamond Urbano, Yoon-Hee Cha and Lei Ding. ICA on sensor or source data: A comparison study in deriving resting state networks from EEG. Engineering in Medicine and Biology Society (EMBC), 2017, 39th Annual International Conference of the IEEE, 14 September 2017.

[15] Munyaradzi C. Rushambwa and Asaithambi Mythili. Impact assessment of mental subliminal activities on the human brain through neuro feedback analysis. 3rd International Conference on Biosignals, images and instrumentation (ICBSII), 16-18 March 2017, Chennai.

[16] Jalal Karam, Salah Al Majeed, Christofer N. Yalung and Lela Mirtskhulava. Neural network for recognition of brain wave signals. International Journal of Enhanced Research in Science, Technology \& Engineering, 2016, Vol. 5 Issue 10, ISSN: 2319-7463.

[17] David C Jangraw, Jun Wang, Brent J Lance, Shih-Fu Chang, and Paul Sajda. Neurally and ocularly informed graph-based models for searching 3D environments, J. Neural Eng. 11 (2014) 046003 (12pp).

[18] Sabbir Ibn Arman, Arif Ahmed, and Anas Syed. Costeffective EEG signal acquisition and recording system. International Journal of Bioscience, Biochemistry and Bioinformatics, 2012, 2 (5):301-304.

[19] Durgesh K. Srivastava and Lekha Bhambhu. Data classification using support vector machine. Journal of Theoretical and Applied Information Technology, 2010, 12 (1): 1-7.

[20] Himani Bhavsar and Mahesh H. Panchal. A review on support vector machine for data classification. International Journal of Advance Research in Computer Engineering \& Technology (IJARCET), 2012, Volume 1, Issue 10, ISSN: 2278-1323.

[21] Adrian G. Bors. Introduction of the radial basis function (RBF) networks. Online Symposium for Electronics Engineers, 2001, 1 (1):1-7.

\section{Authors' Profiles}

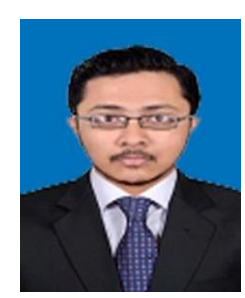

Rajib Chowdhury received B.Sc. in Electrical and Electronic Engineering from Faculty of Engineering, American International University Bangladesh (AIUB), Dhaka, Bangladesh in January 2013. Currently, he is a student of Master of Science in Computer Science (Intelligent Systems) at Faculty of Information Science and Technology, American International University Bangladesh (AIUB), Dhaka, Bangladesh. His research interests include Image Processing, Computer Vision, Artificial Intelligence, 
Augmented Reality, 3D Reconstruction, and Medical Image Processing.

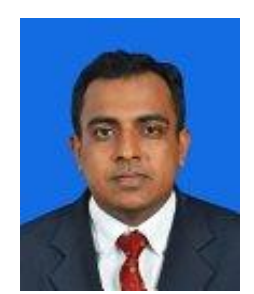

A.F.M. Saifuddin Saif received Ph.D. from Faculty of Information Science and Technology, University Kebangsaan Malaysia (UKM) in 2016. He received M.Sc. in Computer System Engineering (Software System) from University of East London, UK, and B.Sc. (Eng.) degree in Computer Science and Engineering from Shahjalal University of Science and Technology, Bangladesh in 2012 and 2008, respectively. Most of his contributions in Computer Vision and Artificial Intelligence Research field were published in ISI Q1 journals. He has published many papers in ISI indexed Journals; Scopus indexed Journals, Book Chapters, Conferences, and Proceedings. He served as Technical Committee Members, Reviewers, Guest Speakers, Session Chairs in many Conferences and Workshops. Currently, he is an Assistant Professor at Faculty of Information Science and Technology, American International University Bangladesh (AIUB). Before joining the university, he did Post Doctorate at Faculty of Information Science and Technology, University Kebangsaan Malaysia. He spent more than 6 years in IT industry such as Advanced Software Development, Web eMaze etc as IT researcher. His research interests include Image Processing, Computer Vision, Artificial Intelligence, Augmented Reality, 3D Reconstruction, and Medical Image Processing.

How to cite this paper: Rajib Chowdhury, A.F.M. Saifuddin Saif, " Efficient Mathematical Procedural Model for Brain Signal Improvement from Human Brain Sensor Activities ", International Journal of Image, Graphics and Signal Processing(IJIGSP), Vol.10, No.10, pp. 46-53, 2018.DOI: 10.5815/ijigsp.2018.10.05 